(C) 2001 IEEE. Personal use of this material is permitted. Permission from IEEE must be obtained for all other uses, in any current or future media, including reprinting/republishing this material for advertising or promotional purposes, creating new collective works, for resale or redistribution to servers or lists, or reuse of any copyrighted component of this work in other works. 


\title{
Understanding the Effects of Phase Noise in Orthogonal Frequency Division Multiplexing (OFDM)
}

\author{
Ana García Armada, Member, IEEE
}

\begin{abstract}
Phase noise must be carefully considered when designing an OFDM-based communication system since an accurate prediction of the tolerable phase noise can allow the system and RF engineers to relax specifications. This paper analyzes the performance of OFDM systems under phase noise and its dependence on the number of sub-carriers both in the presence and absence of a phase correction mechanism. Besides some practical results are provided so as to give some insight into the phase noise spectral specifications that should be required to the local oscillator.
\end{abstract}

Index Terms-Multicarrier modulation, OFDM, phase noise.

\section{INTRODUCTION}

$\mathbf{O}$ FDM (Orthogonal Frequency Division Multiplexing) is a multicarrier modulation that is implemented in many recent wireless applications due to its ability to combat impulsive noise and multipath effects and make better use of the system available bandwidth. It has been adopted for the European Digital Audio Broadcasting (DAB) [1] and Digital Video Terrestrial Broadcasting (DVB) [2] standards, it has been proposed for UMTS (Universal Mobile Telecommunication Systems) [3] and it has just been standardized for new wireless LAN generations (HIPERLAN: High Performance Radio LAN) [4].

Phase noise must be carefully considered when dealing with any of these communication systems since an accurate prediction of the tolerable phase noise can allow the system and RF designers to relax specifications. This fact is of special importance in those systems that are transmitting OFDM signals at frequencies above $25 \mathrm{GHz}$, as suggested in some European ACTS projects dealing with LMDS (Local-Multipoint Distribution Systems) [5], because of the state-of-the-art RF technology involved.

Phase noise effects in OFDM have been analyzed by several authors and the degradation introduced in the system by phase noise has been characterized for some particular [6]-[9].

The analysis of [6] is somehow optimistic since it is assumed that the constant phase error is ideally corrected. Results will be presented here that consider a particular correction scheme.

It is demonstrated in [7] that the degradation caused in OFDM by phase noise is the same as in a single carrier system when phase noise effects are considered without attempting to correct

This work was supported in part by the European Commission and Spanish CICYT in the project 2FD97-1066-C02-02. them. It will be shown in this paper that this does not hold if phase noise effects are corrected.

Some kind of channel correction mechanism is generally introduced in OFDM systems, what can also correct phase noise to some extent. This fact explains the importance of being able to predict the performance of an OFDM system when this correction is applied.

Previous studies of phase noise in OFDM analyze the system performance dependence with some phase noise parameters, such as the 3-dB linewidth of Lorentzian power density [8] or the correlation time [9]. However, these are not easily related to the oscillator specifications needed by the system designers.

The purpose of this paper is to analyze the performance of OFDM systems under phase noise and to study its dependence on the number of sub-carriers both in the presence and absence of a phase correction mechanism. Besides some practical results will be provided so as to give some insight into the phase noise spectral specifications that should be required to the local oscillator.

This paper is organized as follows. After a brief introduction to OFDM systems (Section II), phase noise is reviewed and a phase noise model for simulation purposes is introduced (Section III). Phase noise effects on the OFDM signal are analyzed in Section IV both theoretically and via simulation. Finally, some conclusions follow.

\section{ORTHOgOnAL FREQUENCY Division MultiPleXING} (OFDM)

Orthogonal Frequency Division Multiplexing (OFDM) constitutes one of the best alternatives to alleviate multipath effects in mobile communications [10]. For reception in built-up areas with delay spreads of some microseconds, the channel is highly frequency selective and sophisticated equalization techniques are required to achieve high bit rate transmissions. However, a combination of OFDM and coding associated with interleaving in the frequency domain (COFDM) can take advantage from the diversity associated to multipath [11].

In an OFDM system the spectrum associated to each elemental data is a small portion of the total bandwidth $(B)$, which is divided in $N$ sub-channels. Each of them is modulated with one information symbol and they are all multiplexed in frequency.

If $T$ represents the OFDM symbol duration, that is: $T=$ $N / B$, the $N$ sub-carriers are placed in the frequencies:

$$
f k=f o+k / T, \quad k=0,1, \ldots, N-1 .
$$


The different sub-channels are allowed to overlap so as to increase the bandwidth efficiency.

When sampled with sampling frequency $f_{s}=N / T$, the complex envelope of the OFDM signal can be obtained by an inverse Discrete Fourier Transform [12] of the complex data sequence $s_{i k}$ (where $i$ is the time index and $k$ is the sub-carrier index):

$$
x(n)=\sum_{i=-\infty}^{\infty} \operatorname{DFT}^{-1}\left\{s_{i k}\right\} \Pi_{N}(n-i N) .
$$

Here $\Pi_{N}(n)$ represents a rectangular pulse with $N$ samples of duration.

The demultiplexing process can be accomplished by means of a Discrete Fourier Transform in the receiver [12], which guarantees orthogonallity and simplifies the design.

Usually a Guard Interval is added to the signal obtained by IDFT in the transmitter so as to cope with inter-symbol interference (ISI) caused by multipath channels. It consists of a repetition of a certain number of the last samples of the useful symbol that are added at its beginning (commonly known as the Cyclic Prefix).

Two Frequency Guards are also inserted, leaving a group of frequencies at the lower and upper parts of the available bandwidth without modulation, in order to avoid adjacent channel interference (ACI).

The advantages of this parallel data transmission with several sub-channels carrying low rate information were suggested by S. B. Weinstein and P. M. Ebert in 1971 [12] and later by L. J. Cimini in 1985 [10]. Because each sub-channel covers only a small portion of the whole bandwidth, equalization is potentially easier than in serial transmission systems, and it can take advantage of the inclusion of pilot sub-carriers in the frame [10].

The estimation of channel attenuation from known symbols multiplexed in the frame was introduced by Moher and Lodge [13] for single carrier systems under the name PSAM (pilot-symbol assisted modulation) and analyzed by Cavers [14]. The extension to multicarrier systems implies considering a two-dimensional space (time and frequency) with an increase in complexity that can be reduced if both dimensions are separated.

B. Le Floch, M. Alard and C. Berrou have recently considered this kind of pilot-based equalization for the broadcasting of COFDM digital television [11]. Although the European DVB-T standard specifies only the signal transmission [2], several pilot sub-carriers have been defined so as to provide a means for channel correction in the receiver.

This same correction scheme that is intended for correcting the multipath channel effects, can also be used to compensate the distortion caused by phase noise.

\section{Phase NoISE}

Phase noise effects introduced by the local oscillator in any receiver can only be ameliorated by improving the performance of the oscillator itself, with the associated cost increase. Hence the importance of determining how much phase noise a receiver can withstand while maintaining the required performance.

Phase noise can be interpreted as a parasitic phase modulation in the oscillator's signal, which ideally would be a unique carrier with constant amplitude and frequency. It has been modeled for simulation purposes as a phase modulation of the carrier.

The modulating signal is a zero mean white Gaussian random process $\phi_{W}(n)$, with variance $\sigma_{W}^{2}$. Its auto-correlation function is given by:

$$
R_{\phi_{W}}(k)=\sigma_{W}^{2} \cdot \delta(k) .
$$

And its power spectral density:

$$
S_{\phi_{W}}(f)=\sum_{k=-\infty}^{\infty} R_{\phi_{W}}(k) \cdot e^{-j 2 \pi f k}=\sigma_{w}^{2} .
$$

It has been low pass filtered with an impulse response $h_{L P F}(n)$, so that its power spectral density becomes:

$$
S_{\phi}(f)=S_{\phi_{W}}(f) \cdot\left|H_{L P F}(f)\right|^{2} .
$$

In this way we can analyze the influence of the phase noise bandwidth $B_{\phi}$ (given by $3 \mathrm{~dB}$-bandwidth of the filter) and its correlation characteristics in the signal quality.

We have found it useful to compare this phase noise bandwidth and the OFDM inter-carrier spacing $\Delta f=B / N$, where $N$ is the number of OFDM sub-carriers and $B$ the total available bandwidth. We may also compare the reciprocal of the filtered phase noise bandwidth and the OFDM symbol duration [9].

The phase noise variance after filtering $\sigma^{2}$ (in radians ${ }^{2}$ ) is also used as a parameter that will be easy to relate to commonly used phase noise specifications as shown later in this paper.

\section{Phase Noise Effects IN The Quality OF AN OFDM SIGNAL}

Phase noise effects in the quality of an OFDM signal will be approached both from a theoretical point of view and simulations. The theoretical analysis will allow us to identify two different kinds of effects that are introduced by phase noise in OFDM signals: common phase error and inter-carrier interference. Simulations will provide error performance results in nonideal situations.

The assumptions and simplifications (small phase noise values) made in the analysis to understand the system behavior have not been applied to simulations, so results can be obtained for any phase noise and channel situations.

In the following sections phase noise effects in OFDM and single carrier (SC) signals, tolerable phase noise spectrum masks and improvements achieved by phase noise correction are analyzed.

\section{A. Theoretical Analysis of Phase Noise}

A theoretical analysis of phase noise effects in OFDM signals can be found in [9]. In order to understand the meaning of the different parameters, this analysis is reproduced here without details:

Taking into account (2), the complex envelope of the transmitted OFDM signal for a given OFDM symbol, sampled with sampling frequency $f_{s}=B$, is:

$$
x(n)=\sum_{k=0}^{N-1} s_{k} \cdot e^{j(2 \pi / N) k n}
$$


with $n=0,1, \ldots, N-1$.

This symbol is actually extended with a Time Guard in order to cope with multipath delay spread, as explained in Section II. For the sake of simplicity, we will not consider this prefix since it is eliminated in the receiver.

Assuming that the channel is flat, the signal is only affected by phase noise $\phi(n)$ at the receiver:

$$
r(n)=x(n) \cdot e^{j \phi(n)} .
$$

The received signal is Orthogonal Frequency Division Demultiplexed (OFDD) by means of a Discrete Fourier Transform. In order to separate the signal and noise terms, let us suppose that $\phi(n)$ is small, so that:

$$
e^{j \phi(n)} \approx 1+j \phi(n) .
$$

In this case, the demultiplexed signal is:

$$
\begin{aligned}
& y(k) \approx s_{k}+\frac{j}{N} \sum_{r=0}^{N-1} s_{r} \sum_{n=0}^{N-1} \phi(n) \cdot e^{j(2 \pi / N)(r-k) n} \\
& y(k) \approx s_{k}+e_{k} .
\end{aligned}
$$

Thus we have an error term $e_{k}$ for each sub-carrier which results from some combination of all of them and is added to the useful signal. Let us analyze more deeply this noise contribution:

1) If $r=k$ : Common Phase Error:

$$
\frac{j}{N} \sum_{r=0}^{N-1} s_{r} \sum_{n=0}^{N-1} \phi(n)=j \cdot s_{k} \cdot \Phi .
$$

We have a common error added to every sub-carrier that is proportional to its value multiplied by a complex number $j \Phi$, that is a rotation of the constellation.

This angle results from an average of phase noise:

$$
\Phi=\frac{1}{N} \sum_{n=0}^{N-1} \phi(n) .
$$

This average implies low frequencies of phase noise spectrum up to $B / N$, that is the inter-carrier spacing $\Delta f$. Since it is constant for all sub-carriers, it can be corrected by some kind of phase rotation.

2) If $r \neq k$ : Inter-Carrier Interference:

$$
\frac{j}{N} \sum_{\substack{r=0 \\ r \neq k}}^{N-1} s_{r} \sum_{n=0}^{N-1} \phi(n) \cdot e^{j(2 \pi / N)(r-k) n} .
$$

This term corresponds to the summation of the information of the other $N-1$ sub-carriers each multiplied by some complex number which comes from an average of phase noise with a spectral shift. The result is also a complex number that is added to each sub-carrier's useful signal and has the appearance of Gaussian noise. It is normally known as inter-carrier interference (ICI) or loss of orthogonallity [15], [16].

The spectral components of phase noise that contribute to the magnitude of this error are those from $\Delta f$ up to the total

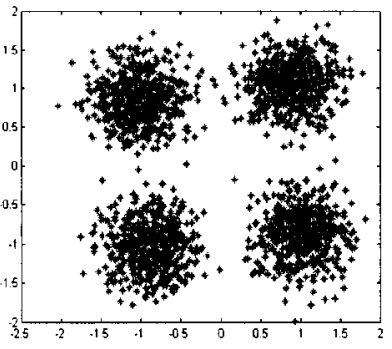

(a)

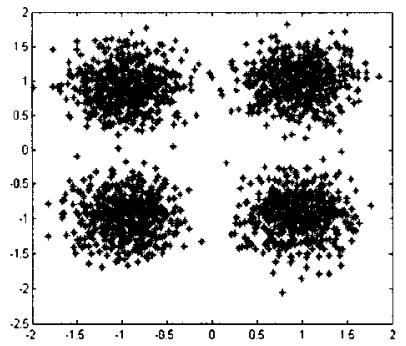

(b)

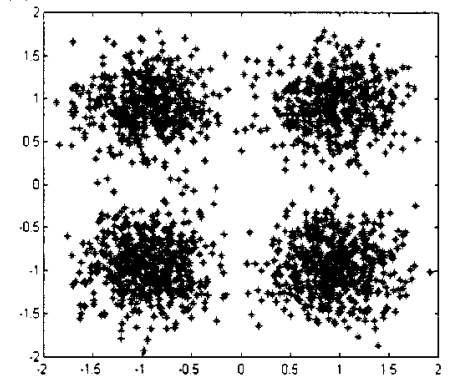

(c)

Fig. 1. Phase noise effects in the OFDD signal.

phase noise bandwidth. Because of its random nature, it cannot be corrected.

Fig. 1 shows the combination of these two effects on a QPSK-OFDM signal after demodulation (OFDD). The first scatter plot (a) corresponds to the OFDD signal when the variance of the phase noise that affects the OFDM signal is $\sigma^{2}=0.1 \mathrm{rad}^{2}$ and the relationship of phase noise bandwidth and inter-carrier spacing is $B_{\phi} / \Delta f=0.01$. A rotation of the constellation and some inter-carrier interference (appearance of white noise clouds) can be appreciated.

Since this rotation (common phase error) is identical for all the sub-carriers, the phase difference between consecutive symbols can be obtained with the aid of pilots introduced in the OFDM frame and corrected [9].

After removing the common phase error in the signal Fig. 1(a), the constellation looks as shown in Fig. 1(b).

When the phase noise bandwidth is greater, inter-carrier interference (ICI) dominates over the common phase error. This situation is shown in Fig. 1(c) in which the same phase noise variance has been introduced but $B_{\phi} / \Delta f=1$. It can be seen that ICI has increased and constellation rotation is not perceptible anymore.

\section{B. Phase Noise in OFDM and Single Carrier Systems}

It is shown in [7] that the signal to noise ratio degradation $\left(\operatorname{deg}_{\mathrm{SNR}}\right)$ caused by phase noise is the same in OFDM and SC systems and, given that phase noise variance is small $\left(\sigma^{2} \ll 1\right)$, it follows the expression:

$$
d e g r_{\mathrm{SNR}}=10 \cdot \log \left(1+\sigma^{2} \frac{E_{s}}{N_{0}}\right) \mathrm{dB} .
$$

In this equation $E_{s}$ represents the symbol energy and $N_{0}$ is the power spectral density of additive white Gaussian noise.

Simulations that have been run for an OFDM signal with 2048 (2K) sub-carriers modulated in QPSK confirm this formula. The 


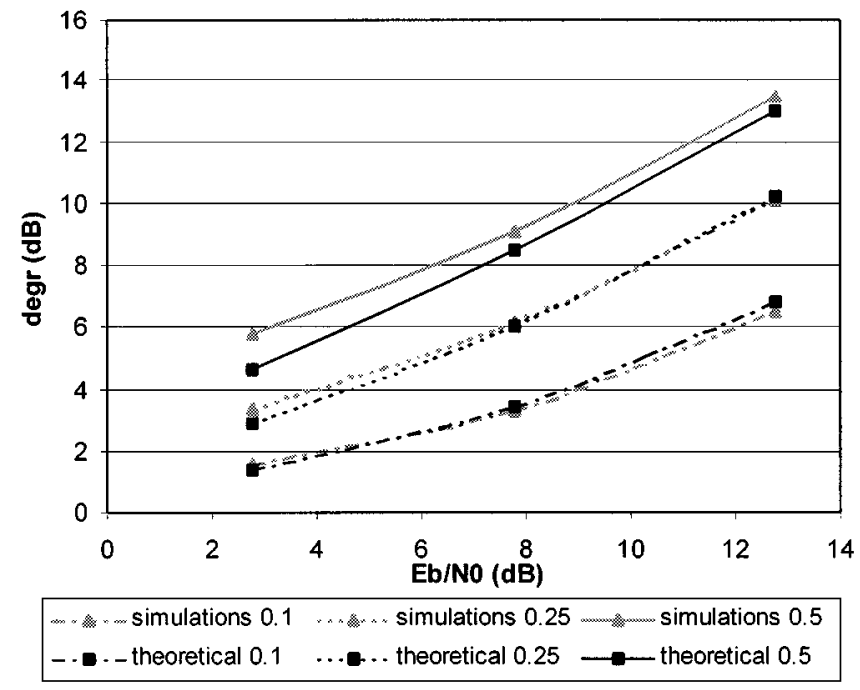

Fig. 2. Phase noise degradation in QPSK-OFDM.

symbol error probability (SER) has been estimated for different values of the phase noise variance $\left(\sigma^{2}\right)$ and signal to noise ratios $\left(E_{b} / N_{0}\right.$ Fig. 2 shows some results.

For simulations of Fig. 2 the lower 171 and upper 172 frequencies have been left without modulation (Frequency Guards) and a Guard Interval equal to $T / 8$ ( $T$ equals the OFDM symbol duration) has been used. However, the same results are obtained independently of the values chosen for these parameters.

From inspection of Fig. 2 we can see that the smaller the phase noise variance, the closer the simulation results to theoretical values of (14).

Besides, simulations show that the degradation in OFDM does not depend on the number of sub-carriers and phase noise bandwidth, as stated in expression (14).

\section{Tolerable Phase Noise Spectrum Masks in OFDM Systems}

The most common way of characterizing oscillator's phase noise is the single-side-band phase noise power density function $L\left(f_{m}\right)$, which represents the ratio (in $\mathrm{dBc}$; ' $\mathrm{c}$ ' stands for carrier) between the single-side-band noise power in a $1 \mathrm{~Hz}$ bandwidth at a distance $f_{m}$ from the carrier and the carrier power [17].

This characterization is normally performed by using a spectrum analyzer which provides the power spectral density of the equipment's phase noise $\left(N_{0 p}\right)$ in relation to the carrier power $(C)$. From these measurements, given that the phase noise has a zero mean as we have assumed and it extends up to a frequency $b$ (either because phase noise is band-limited or due to the presence of filtering in the receiver), its variance can be found as [17]:

$$
\sigma^{2}=\int_{0}^{b}\left(\frac{2 N_{0 p}}{C}\right) d f
$$

Once that its variance can be found, expression (14) provides the SNR degradation introduced by phase noise.

Figs. 3 and 4 show examples of typical phase noise spectrum masks: spectrum of Fig. 3 may be easy to achieve with not very expensive components while spectrum of Fig. 4 can only be obtained by changing the components of the oscillator for some

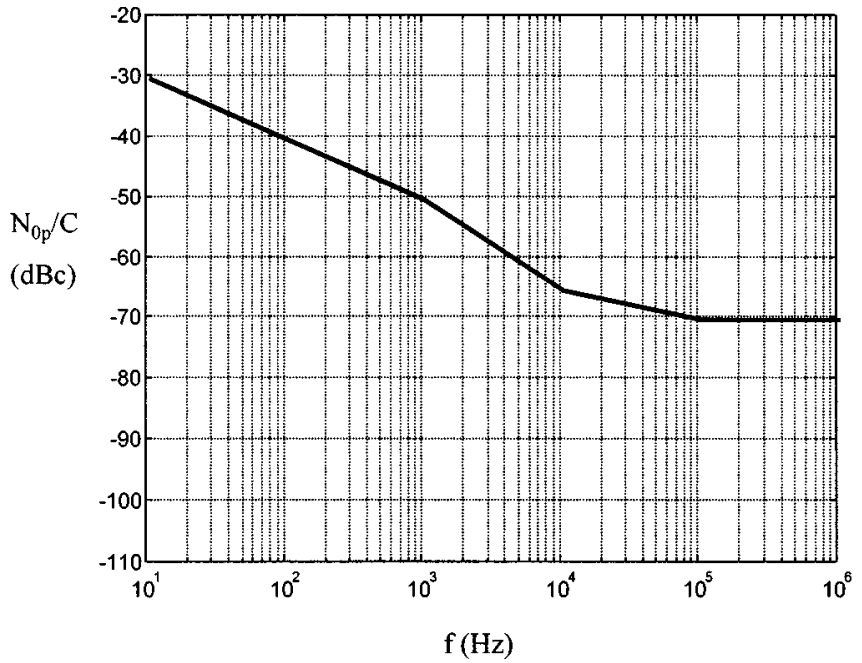

Fig. 3. Phase noise spectrum mask: $\sigma^{2}=1.33 \mathrm{rad}^{2}$.

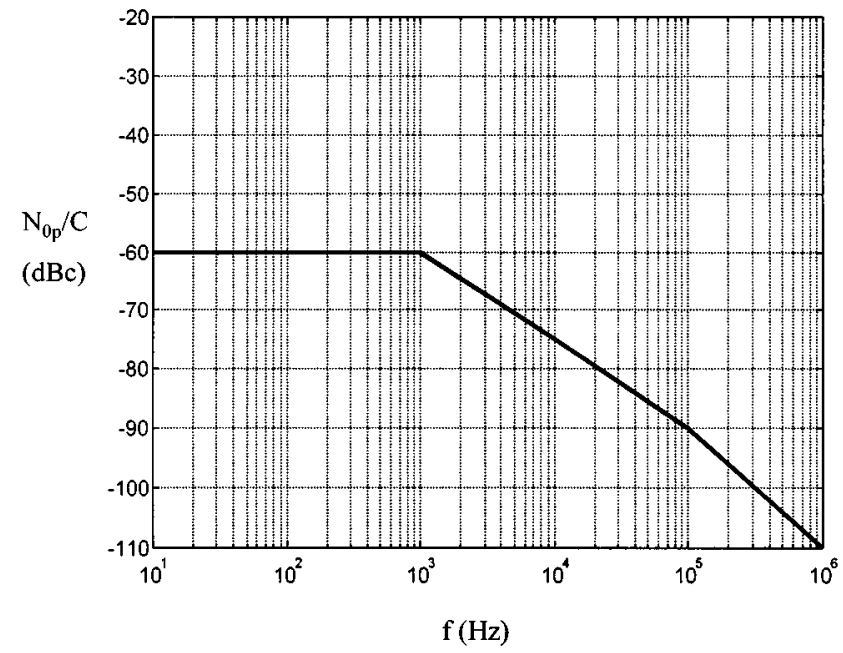

Fig. 4. Phase noise spectrum mask: $\sigma^{2}=0.015 \mathrm{rad}^{2}$.

better and more expensive ones (note the decrease in the noise floor at high frequencies). Phase noise variance values are respectively $\sigma^{2}=1.33 \mathrm{rad}^{2}$ and $\sigma^{2}=0.015 \mathrm{rad}^{2}$.

The performance of an OFDM system corrupted with phase noise has been estimated via simulations for these two variance values. In the first case, since the variance is not small enough, simulations are needed in order to obtain accurate results. In the second case, simulated and theoretically predicted degradations coincide.

The OFDM signal has again $2 \mathrm{~K}$ sub-carriers and the same parameters as in the system simulated in Section IV-B.

The SNR degradation caused by phase noise in the two cases described above is presented in Fig. 5. As it may have been expected, the degradation is much higher in the system corrupted with the phase noise of highest variance.

Since a phase noise variance of $1.33 \mathrm{rad}^{2}$ causes degradations of values between 10 and $25 \mathrm{~dB}$ for commonly used $E_{b} / N_{0}$ ratios, we can conclude that an oscillator with the phase noise mask shown in Fig. 3 should not be used in an OFDM equipment.

In the second case, however, the degradation is much lower. Moreover, it can be neglected for $E_{b} / N_{0}$ values below $48 \mathrm{~dB}$, 


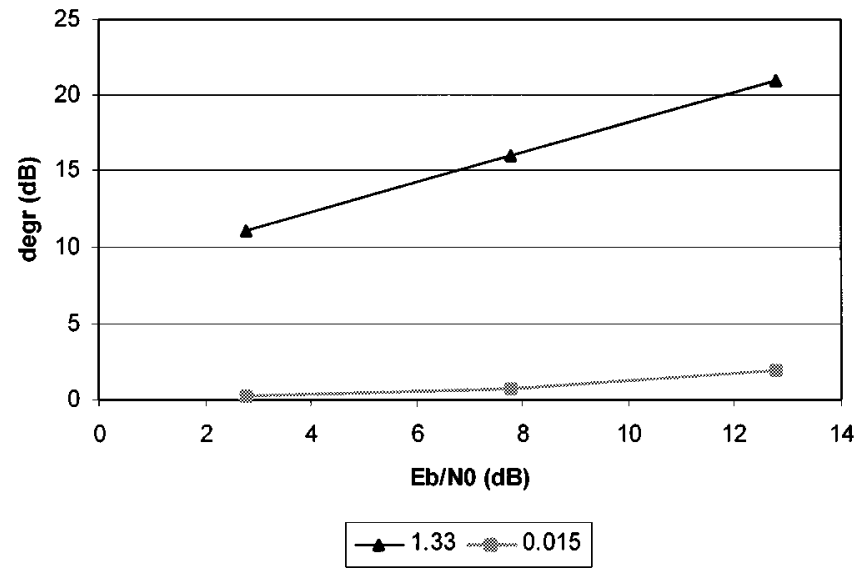

Fig. 5. SNR degradation for $\sigma^{2}=1.33$ and $\sigma^{2}=0.015$.

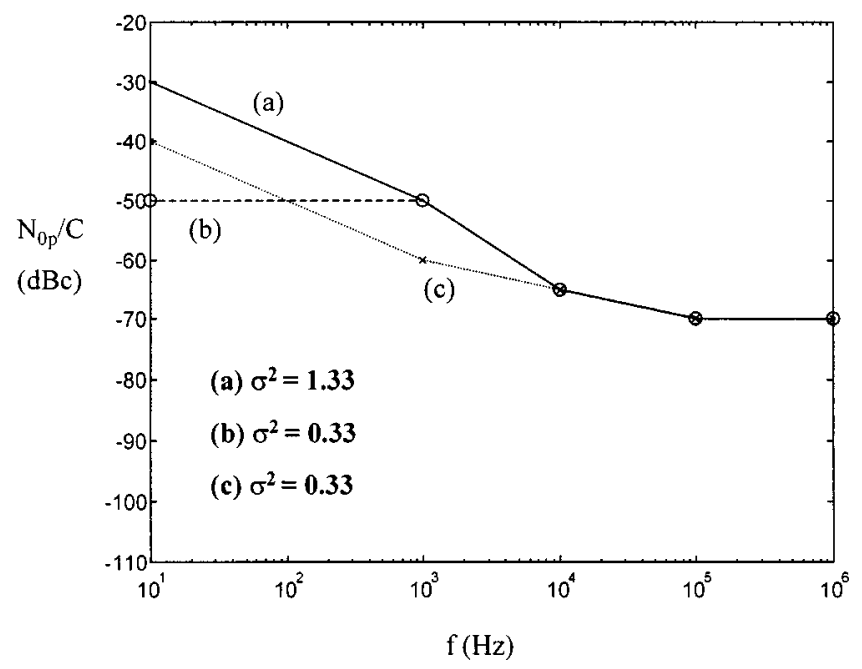

Fig. 6. Alternative phase noise masks.

what leads us to the conclusion that the second phase noise mask would be very well tolerated by an OFDM system.

In an attempt to achieve better results with the first oscillator one might improve it by lowering the phase noise power in the region of the spectrum close to the carrier. This can be done with a careful adjust of the PLL filter. Two alternatives are shown in Fig. 6 in which (a) represents the same spectrum mask as in Fig. 3, (b) shows one possible power reduction and (c) another one.

However, in both (b) and (c) phase noise variance is only reduced to $0.33 \mathrm{rad}^{2}$, causing a SNR degradation between 5 and $12 \mathrm{~dB}$, not low enough.

This example illustrates the importance of the higher frequencies of the phase noise spectrum. It is clear that the whole phase noise power spectrum must be lowered in order to achieve an adequate performance.

\section{Performance After Common Phase Error Correction}

Although the degradation caused by phase noise is independent of the number of sub-carriers in the OFDM signal and the phase noise bandwidth, as shown in the preceding sections, the situation is different when we try to correct phase noise effects.

The only phase noise effect that can be corrected is the common phase error. Since its magnitude depends on the phase

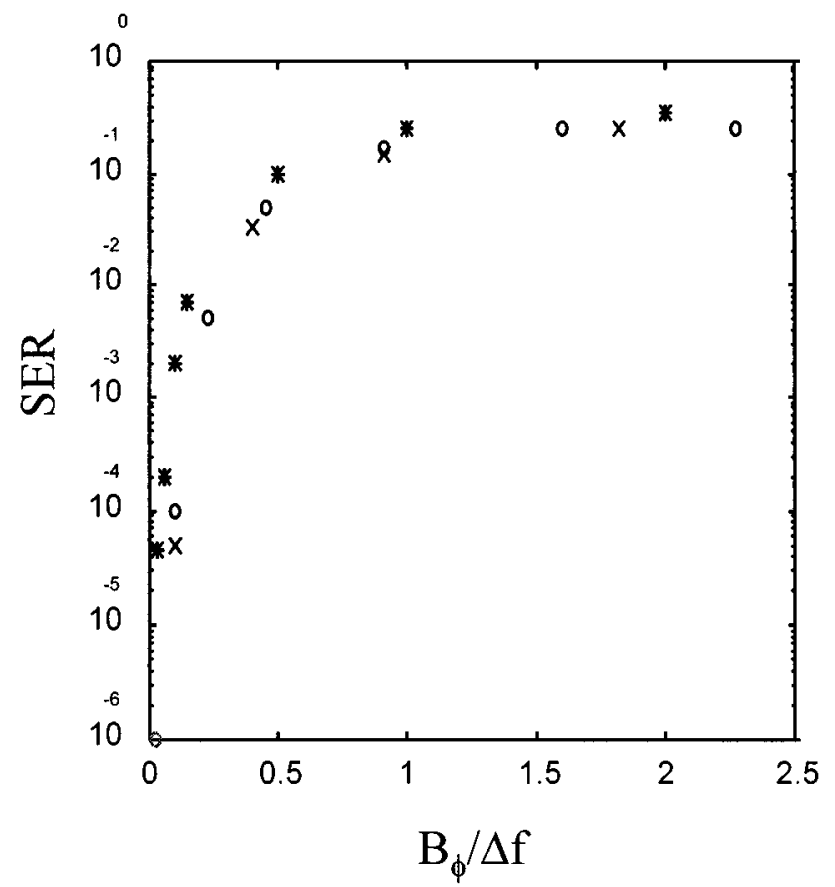

Fig. 7. SER after correction with different numbers of sub-carriers: $8 \mathrm{~K}(x)$, $2 \mathrm{~K}(\mathrm{o})$ and $128(*)$.

noise bandwidth as explained in Section IV-A and illustrated Fig. 1, the same happens to the correction capabilities.

This common phase error can be removed by any phase correction scheme. The results presented in this paper have been obtained taking advantage of the pilot-based correction mechanism that is implemented to correct the channel effects in OFDM systems like DVB-T [2]. More details about this correction scheme can be found in [18].

When the ratio between the phase noise bandwidth $B_{\phi}$ and the OFDM inter-carrier spacing $\Delta f$ is small, it comes out from the discussion on Section III that the common phase error dominates over the inter-carrier interference (ICI). Since this error can be corrected together with the channel effects [9], the symbol error rate after correction is much lower than before as it will be shown in the following.

On the other hand, as this ratio approaches unity, that is, the phase noise bandwidth becomes closer to the inter-carrier spacing value, the inter-carrier interference increases and the correction capabilities decrease. If $B_{\phi}$ is greater than $\Delta f$, the symbol error rate after correction can be equal to that obtained before correction or even worse if the chosen phase correcting scheme fails due to the presence of noise.

If we consider the phase noise bandwidth normalized to the OFDM inter-carrier spacing $\Delta f$, the symbol error rate is independent of the number of sub-carriers. However, there may be a slight difference if the phase correction system improves with the number of sub-carriers as in our case [9].

This situation is illustrated in Fig. 7 showing the symbol error rate (SER) obtained when the number of sub-carriers varies and signal-to-noise ratio and phase noise variance are fixed.

The situation is different if the phase noise bandwidth $B_{\phi}$ is considered without normalization. Since the inter-carrier spacing value $\Delta f$ depends on the number of sub-carriers in the OFDM signal, the limit situation $B_{\phi} / \Delta f=1$ is achieved in a 


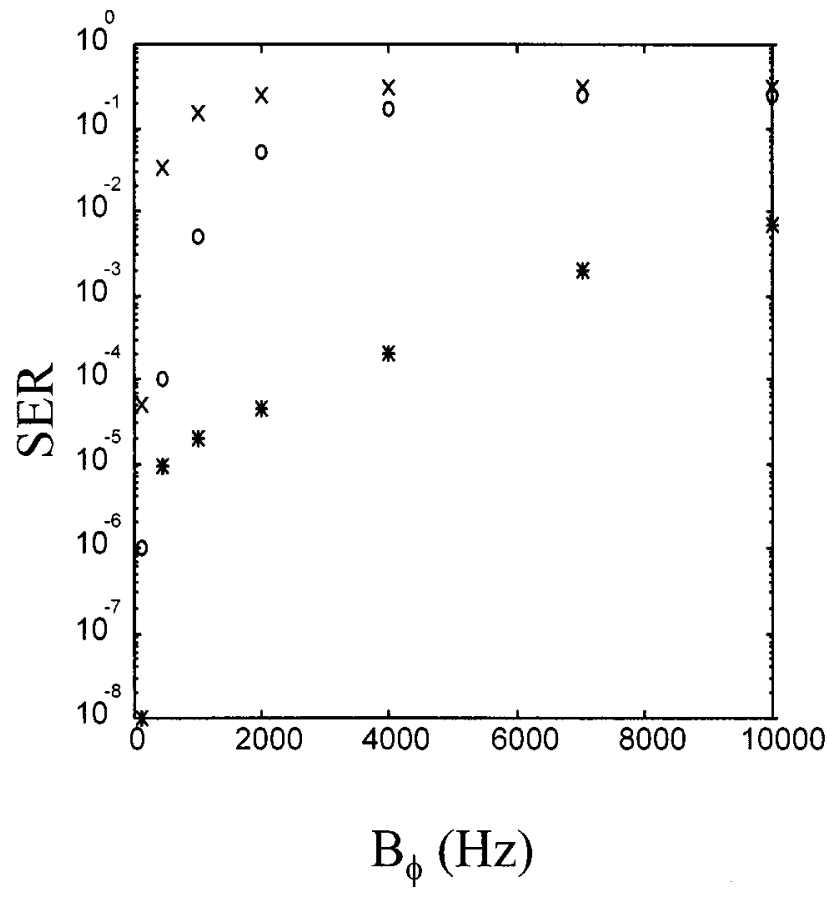

Fig. 8. SER after correction and $B_{\phi}(\mathrm{Hz})$ with different numbers of sub-carriers: $8 \mathrm{~K}(\times), 2 \mathrm{~K}(\mathrm{o})$ and $128(*)$.

system with many sub-carriers for a given phase noise when we still have $B_{\phi} / \Delta f \ll 1$ in a system with much less sub-carriers.

Results of Fig. 7 are presented in Fig. 8 with phase noise bandwidth expressed in $\mathrm{Hz}$.

Only if the phase noise bandwidth is greater than the intercarrier spacing for the three compared systems $(8 \mathrm{~K}, 2 \mathrm{~K}$ and 128 sub-carriers), their performance after correction will be the same, because the ICI will dominate in all of them and the correction will not be appreciable. If this is not the case, as it should be expected since it is desirable for the correcting scheme to be able to function properly, the system with the lowest number of sub-carriers will exhibit the best error performance.

\section{E. SNR Degradation After Common Phase Error Correction}

The SNR degradation introduced by phase noise after correction has been evaluated via simulation and is presented here so that it can be compared with the values obtained without correction in Sections IV-B and IV-C.

Simulations have been run for an OFDM system with $2 \mathrm{~K}$ subcarriers and the same frequency and time guards as in the former sections. Common phase error has been corrected as explained in [18].

Fig. 9 shows the degradation that is obtained with and without correction of phase noise effects. Results after correction are only presented for high $E_{b} / N_{0}$ values because the correction scheme's sensitivity to noise would mask the desired results for low $E_{b} / N_{0}$ values.

It can be seen in Fig. 9 that the improvement is more noticeable for high phase noise variances, since the performance without correction is very bad in these cases. For lower values of the phase noise variance there is still a considerable improvement. It can be as high as $5 \mathrm{~dB}$ for $E_{b} / N_{0}$ values around $10 \mathrm{~dB}$.

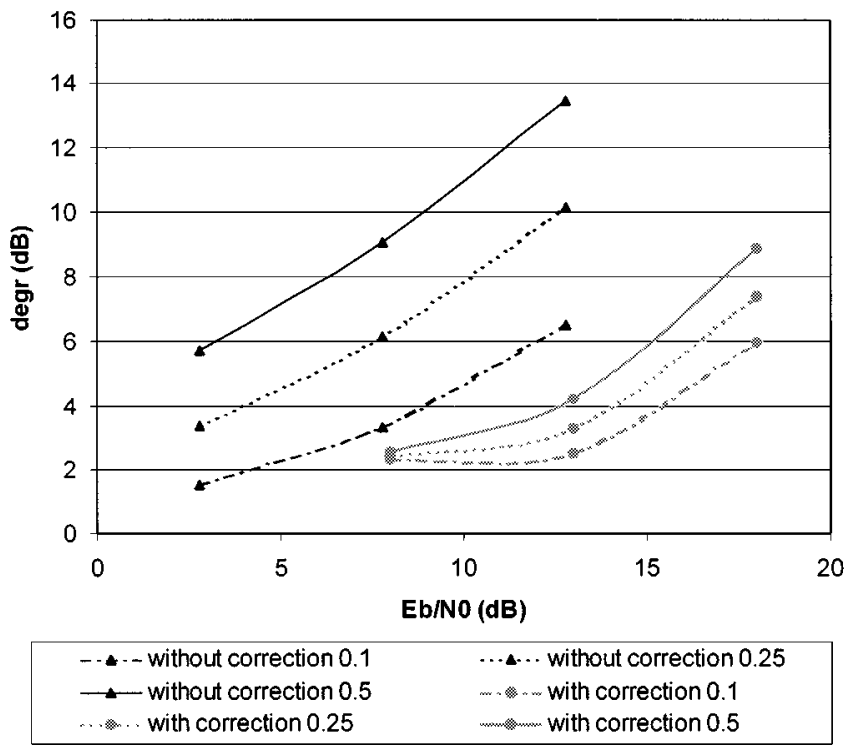

Fig. 9. SNR degradation with phase noise.

If a different correction scheme is used, results may vary depending on the correction capabilities of the adopted mechanism.

In any case, we can conclude that the use of some phase noise correction method allows the oscillator's specifications to be significantly relaxed. For instance, if we recall the example of Fig. 6(b) and (c), we can see now that an oscillator exhibiting one of these phase noise spectrum masks can be used for an OFDM equipment with an appropriate phase correction scheme that provides a $5 \mathrm{~dB}$ gain.

\section{Conclusions}

OFDM (Orthogonal Frequency Division Multiplexing) has been standardized in many recent wireless applications due to its ability to combat impulsive noise and multipath effects and make better use of the system available bandwidth. Since an accurate prediction of the tolerable phase noise can allow the system and RF engineers to relax specifications, an exhaustive analysis of phase noise effects on OFDM signals has been presented.

Theoretical analysis has been contrasted with simulations. The first has allowed us to identify the different kinds of effects that are introduced by phase noise in OFDM signals. Simulations have provided error performance results when phase noise is not small (theoretical results do not apply) or a particular phase error correction scheme is introduced.

It has been shown that the error performance after applying some phase correction scheme depends on the number of sub-carriers that compose the OFDM signal. In most common situations, when the noise bandwidth is not too big compared to the inter-carrier spacing, a system with a lower number of sub-carriers will exhibit a better error performance.

Besides, the error performance has been obtained for different oscillators characterized by their single-side-band phase noise power density functions, giving some insight into the phase noise spectral specifications that should be required to the local oscillator in a practical implementation. 
Finally, it has been concluded that the use of some phase noise correction allows the oscillator's specifications to be significantly relaxed. Since most OFDM systems use some kind of channel correction and the same mechanism can also be used to correct phase noise, it is critical to be able to predict the error performance after correction for different phase noise characteristics.

\section{REFERENCES}

[1] B. Le Floch, R. H. Lassalle, and D. Castelain, "Digital sound broadcasting to mobile receivers," IEEE Trans. Consumer Electronics, vol. 35, no. 3, pp. 493-503, Aug. 1989.

[2] EBU/CENELEC/ETSIT JTC, "Digital broadcasting systems for television, sound and data services, framing structure, channel coding and modulation for digital terrestrial television,”, TM 1545 rev 2, Jan. 1996

[3] J. Van de Beek et al., "On synchronization in an OFDM based UMTS proposal," in Proc. COST 254 (Emergent Techniques for Communication Terminals, Toulouse, France, July 1997.

[4] [Online]. Available: http://www.etsi.fr/bran

[5] [Online]. Available: http://www.uk.infowin.org/ACTS/

[6] T. Pollet, M. van Bladel, and M. Moeneclaey, "BER sensitivity of OFDM systems to carrier frequency offset and Wiener phase noise," IEEE Trans. Commun., vol. 43, no. 2/3/4, pp. 191-193, Feb./Mar./Apr. 1995.

[7] M. Moeneclaey, "The effect of synchronization errors on the performance of orthogonal frequency-division multiplexed (OFDM) systems," in Proc. COST 254 (Emergent Techniques for Communication Terminals), Toulouse, France, July 1997.

[8] L. Tomba, "On the effect of Wiener phase noise in OFDM systems," IEEE Trans. Commun., vol. 46, no. 5, pp. 580-583, May 1998.

[9] A. G. Armada and M. Calvo, "Phase noise and sub-carrier spacing effects on the performance of an OFDM communication system," IEEE Commun. Lett., vol. 2, no. 1, pp. 11-13, Jan. 1998.

[10] L. J. Cimini, "Analysis and simulation of a digital mobile channel using orthogonal frequency division multiplexing," IEEE Trans. Commun., vol. COM-33, no. 7, pp. 665-675, July 1985.
[11] B. Le Floch, M. Alard, and C. Berrou, "Coded orthogonal frequency division multiplex," Proc. IEEE, vol. 83, no. 6, pp. 982-996, June 1995.

[12] S. B. Weinstein and P. M. Ebert, "Data transmission by frequency-division multiplexing using the discrete Fourier transform," IEEE Trans. Commun. Technol., vol. COM-19, no. 5, pp. 628-634, Oct. 1971.

[13] M. L. Moher and J. H. Lodge, "TCMP-A modulation and coding strategy for Rician-fading channels," IEEE J. Sel. Areas Commun., vol. 7, no. 9, pp. 1347-1355, Dec. 1989 .

[14] J. K. Cavers, "An analysis of pilot-symbol assisted modulation for Rayleigh-fading channels," IEEE Trans. Vehicular Technology, vol. 40, no. 4, pp. 686-693, Nov. 1991.

[15] J. H. Stott, "The effect of phase noise in COFDM," EBU Technical Review, Summer 1998.

[16] M. El-Tanany, Y. Wu, and L. Hazy, "Analytical modeling and simulation of phase noise interference in OFDM-based digital television terrestrial broadcasting systems," IEEE Trans. Broadcast., vol. 47, no. 1, Mar. 2001.

[17] W. P. Robins, "Phase noise in signal sources," IEE Telecommunication series $9,1991$.

[18] A. G. Armada and M. Calvo, "Rapid prototyping of a test modem for terrestrial broadcasting of digital television," IEEE Trans. Consumer Electronics, vol. 43, no. 4, pp. 1100-1109, Nov. 1997.

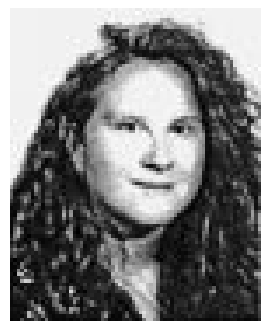

Ana García Armada was born in Santiago de Compostela (Spain) in 1970. She has obtained the S.M. (1994) and Ph.D. (1998) in electrical engineering both from the Polytechnic University of Madrid. She is currently with the Department of Communication Technologies, University Carlos III of Madrid, Spain. Her main interests are in simulation of communication systems and multicarrier modulation techniques. 